\title{
Apathy Measures in Older Adults and People with Dementia: A Systematic Review of Measurement Properties Using the COSMIN Methodology
}

\author{
Clare Burgon ${ }^{a, b}$ Sarah Elizabeth Goldberg ${ }^{a}$ Veronika van der Wardt ${ }^{b, c}$ \\ Catherine Brewin $^{\text {a,d }}$ Rowan H. Harwood ${ }^{a, d}$ \\ aSchool of Health Sciences, University of Nottingham, Nottingham, UK; ${ }^{b}$ Division of Rehabilitation, Ageing and \\ Wellbeing, School of Medicine, University of Nottingham, Nottingham, UK; 'Department of General Medicine, \\ Preventative and Rehabilitative Medicine, Philipps-Universität Marburg, Marburg, Germany; ${ }^{\mathrm{d}}$ Health Care of Older \\ People, Nottingham University Hospitals NHS Trust, Nottingham, UK
}

\section{Keywords}

Apathy - Measurement - Scale - Systematic review .

COnsensus-based Standards for the selection of health

Measurement INstruments

\begin{abstract}
Background: Apathy is highly prevalent in dementia and is also seen in mild cognitive impairment and the general population. Apathy contributes to failure to undertake daily activities and can lead to health problems or crises. It is therefore important to assess apathy. However, there is currently no gold standard measure of apathy. A comprehensive systematic review of the measurement properties of apathy scales is required. Methods: A systematic review was registered with PROSPERO (ID: CRD42018094390). MEDLINE, Embase, PsycINFO, and CINAHL were searched for studies that aimed to develop or assess the validity or reliability of an apathy scale in participants over 65 years, living in the community. A systematic review was conducted in line with the COnsensus-based Standards for the selection of health Measurement INstruments procedure for reviewing patient-reported outcome measures. The studies' risk of bias was assessed, and all relevant measurement properties were assessed for quality. Results were pooled and rated using a modified Grading of Recommendations Assessment, Devel-
\end{abstract}

opment, and Evaluation procedure. Results: Fifty-seven publications regarding 18 measures and 39 variations met the eligibility criteria. The methodological quality of individual studies ranged from inadequate to very good and measurement properties ranged from insufficient to sufficient. Similarly, the overall evidence for measurement properties ranged from very low to high quality. The Apathy Evaluation Scale (AES) and Lille Apathy Rating Scale (LARS) had sufficient content validity, reliability, construct validity, and where applicable, structural validity and internal consistency. Conclusion: Numerous scales are available to assess apathy, with varying psychometric properties. The AES and LARS are recommended for measuring apathy in older adults and people living with dementia. The apathy dimension of the commonly used Neuropsychiatric Inventory should be limited to screening for apathy.

(c) 2021 S. Karger AG, Basel

\section{Introduction}

Apathy is a multidimensional construct, defined as quantitatively reduced behavioural, cognitive, emotional, or social (goal-directed) activity which may include reduced motivation, initiative, effort, interest, concern about self or others, and affect [1]. Apathy is the most karger@karger.com

(c) 2021 S. Karger AG, Basel

www.karger.com/dem

Karger ${ }^{\prime \prime}=$
Correspondence to:

Clare Burgon, clare.burgon@ nottingham.ac.uk 
common neuropsychiatric symptom of dementia [2] and is reported in $15-92 \%$ of people with dementia [3] and $12-40 \%$ of people with mild cognitive impairment (MCI) $[4,5]$. Apathy is associated with important outcomes in dementia and MCI, including disabilities in everyday functioning [6], increased carer burden [7-10], worse adherence to interventions [11,12], and worse quality of life [13]. Prevention or management of apathy in dementia has been identified as a priority area for research [14]. Apathy in older adults is associated with increased likelihood of subsequent cognitive impairment [15], conversion from MCI to dementia $[5,16]$, and worse disease progression $[17,18]$. It is therefore important to research across the spectrum of cognitive impairment [19], including older adults who otherwise appear cognitively unimpaired.

There is no gold standard measure of apathy [20]. Two systematic reviews of apathy scales have been published; the first examined scales developed for people with neurodegenerative conditions such as Parkinson's disease, amyotrophic lateral sclerosis, and dementia [21], whilst the second examined evidence for measurement properties in people with dementia [22]. The first systematic review only included studies that assessed both validity and reliability of a scale within a single publication. Whilst a scale should be both valid and reliable, separately published studies of reliability and validity can collectively offer sufficient evidence for both. The latter review used limited search criteria and it is unclear when the search was conducted. Therefore, important studies regarding the quality of apathy scales may have been missed.

Both of these systematic reviews used the Quality Assessment of Diagnostic Accuracy Studies (QUADAS) tool, designed for studies of diagnostic accuracy [23], not other measurement properties, such as reliability. It is not clear how these reviews rated properties such as reliability using the QUADAS criteria that refer to a "reference standard," which is only relevant to properties such as criterion validity. The COnsensus-based Standards for the selection of health Measurement INstruments (COSMIN) programme of work has since published guidance for conducting and reporting systematic reviews of health measures, with methodological quality standards and measurement property quality criteria that enables a systematic and standardized critical examination of all key measurement properties of scales $[24,25]$. The aim of this study was to assess and compare the quality of measurement properties (i.e., the reliability and validity) and characteristics, of apathy scales and to analyse the quality of the evidence in healthy older adults and people with dementia, in accordance with COSMIN guidance.

\section{Methods}

Design

This systematic review protocol was registered with PROSPERO (ID: CRD42018094390) and published prior to analysis [26]. The COSMIN methodology for conducting systematic reviews of patient-reported outcome measures [24, 25, 27] was followed. Properties were assessed in relation to both people with dementia and older adults. Some additional decisions were required for this review, which were based on literature, discussions with the review team, and PPI input. For example, there is no gold standard time interval for test-retest reliability studies [28] though a time interval of 2 weeks is common [29]. Apathy is a relatively stable, but not an enduring trait, so a time interval that exceeded 28 days or 1 month was considered inappropriate. A time interval of $<3$ days for people with memory problems and $<7$ days for people without memory problems was also considered inappropriate as memory of previous answers may inflate reliability estimates. These were arbitrary numbers chosen in lieu of guidance but was deemed acceptable by the review team and PPI members.

\section{Searching, Screening and Selection}

MEDLINE (In-process, Other nonindexed citations and 1946 onwards), Embase (1980 onwards), PsycINFO (1806 onwards, via Ovid), and CINAHL (1937 onwards) were searched using the specified search strategy on April 18, 2018, and the search was rerun in the same databases on May 6, 2020. The reference lists of the included studies and of any relevant review articles were also examined for relevant publications. The COSMIN search strategy for identifying research on the development, validity, or reliability of health-related outcome measures [30] formed part of the search strategy, along with apathy-related terms (e.g., apathy; lack of or diminished motivation, interest, initiative; emotional blunting; emotional responsiveness; abulia; anhedonia; frontal symptom; asocial; avoliton; and lassitude). The search strategy was first created for MEDLINE (see online suppl. Additional File 1; for all online suppl. material, see www.karger.com/doi/10.1159/000515678; $[99,100,104-107])$, then the subject headings and syntax were adapted for the other databases.

Inclusion criteria: studies that aimed to develop or assess the measurement properties of an apathy scale based on patient or informant reports or interviewer or clinician ratings; primary research; full-text publication; majority of participants living in the community; majority of participants aged 65 or over. Exclusion criteria: studies assessing scales of apathy in a specific context such as work performance or following an event, such as in posttraumatic stress disorder and postnatal depression. Additionally, development studies were included regardless of eligibility criteria if they pertained to an apathy scale that was included in one of the eligible studies.

The titles and abstracts of articles were screened (by $\mathrm{ClB}$ ) to assess whether they met the eligibility criteria. All included full-text articles were assessed against eligibility criteria (by $\mathrm{ClB}$ ), and a randomly selected $10 \%$ of articles were independently assessed by a second reviewer (Ca.B.). Articles for which there was disagreement between reviewers were discussed, and an agreement was reached.

\section{Data Extraction and Assessment}

Data extraction was conducted (by $\mathrm{ClB}$ ) into a data extraction table (online suppl. Additional File 2). Data extraction of $20 \%$ of publications was checked by second reviewers (Ca.B. and V. 
Fig. 1. PRISMA diagram of study selection process.

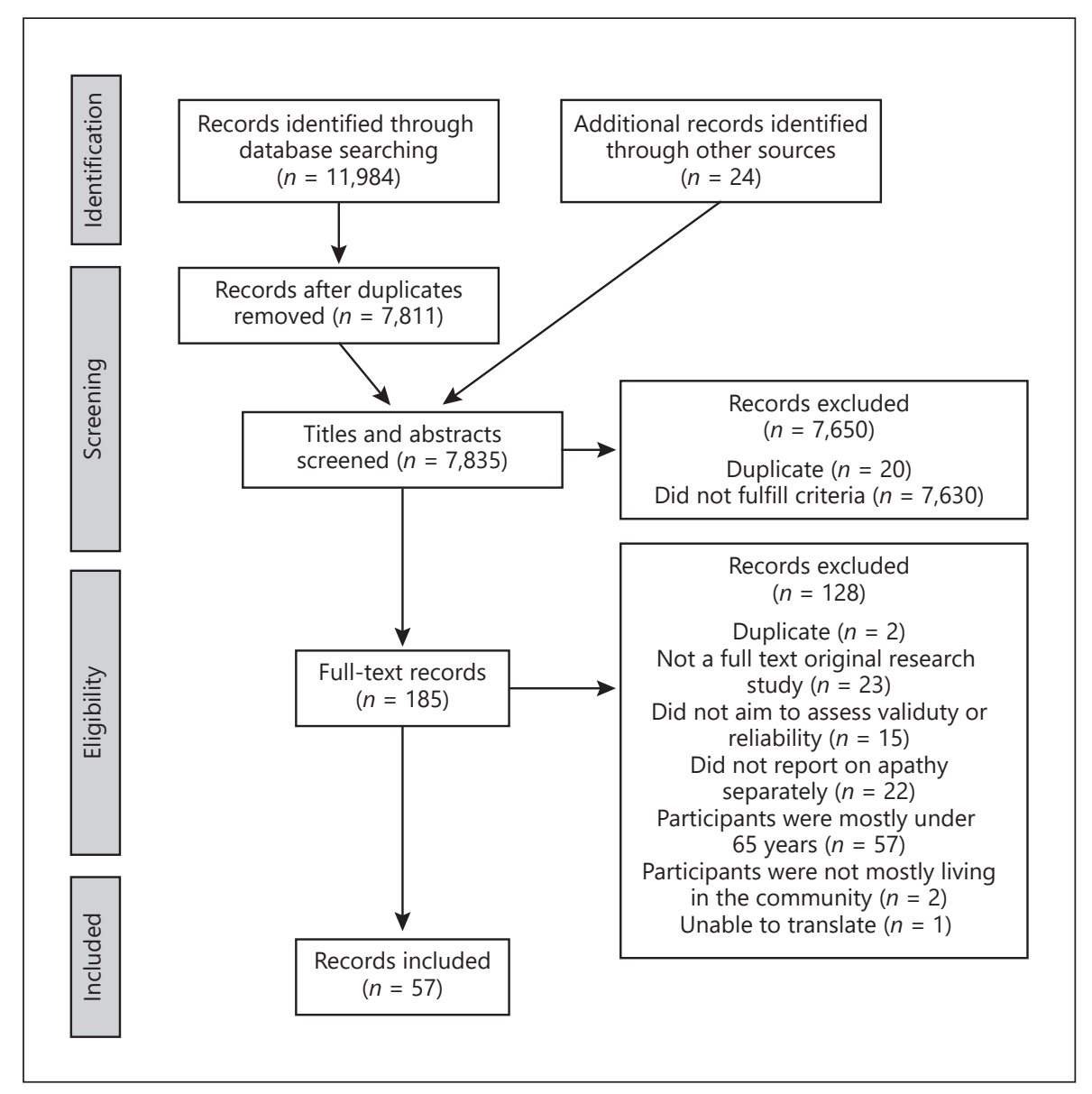

vd.W.), and no errors were found. For each study included in the review, data relating to study characteristics and methods, participant characteristics, and measurement characteristics and properties were extracted. Measurement properties included those of reliability (internal consistency, measurement error, and test-retest and interrater reliability) and validity (content validity, structural validity, and hypothesis testing for construct validity), as defined by the COSMIN taxonomy [31]. Criterion validity and responsiveness were not reviewed, as there is no gold standard measure of apathy against which to assess the scales.

Risk of bias in individual studies was examined using the COSMIN risk of bias checklist [25, 27] (online suppl. Additional File 3). The results of studies were assessed using COSMIN criteria for good measurement properties [24, 25]. Publications were assigned a number and randomly selected for second reviewer ratings using a random number function in Microsoft Excel. Twenty-one percent $(N=$ 12) of publications were independently rated by second reviewers for risk of bias and against criteria for good measurement properties (S.G. and V.vd.W.). Where there was disagreement, this was discussed between the 2 raters (C.l.B. and S.G.; C.l.B. and V.vd.W.) and any remaining disagreements were discussed with a third rater.

\section{Synthesis of Results}

Studies meeting the eligibility criteria were summarized using a narrative synthesis. For each scale, the measurement properties reported in the corresponding studies were summarized, and the quality of these synthesized results was assessed using the criteria for good measurement properties [24, 32]. Where there were different versions of the scale, results were pooled, providing they were not contradictory. The COSMIN-modified GRADE approach $[24,25]$ was used to assess the quality of the cumulative evidence for each measurement property for each scale. The COSMIN procedure for the recommendations of scales in systematic reviews [24] was used to guide the recommendations made.

\section{Results}

\section{Study Selection}

The initial search resulted in 9,645 records and the reexecuted search identified an additional 2,339 records (Fig. 1). Following removal of duplicates, there were 7,811 records. A further 24 records were identified through screening reference lists and manual searching. After screening of titles and abstracts, 185 remained for fulltext screening. Following full-text screening, 57 publications of 18 distinct scales (and 39 variations) were identi- 


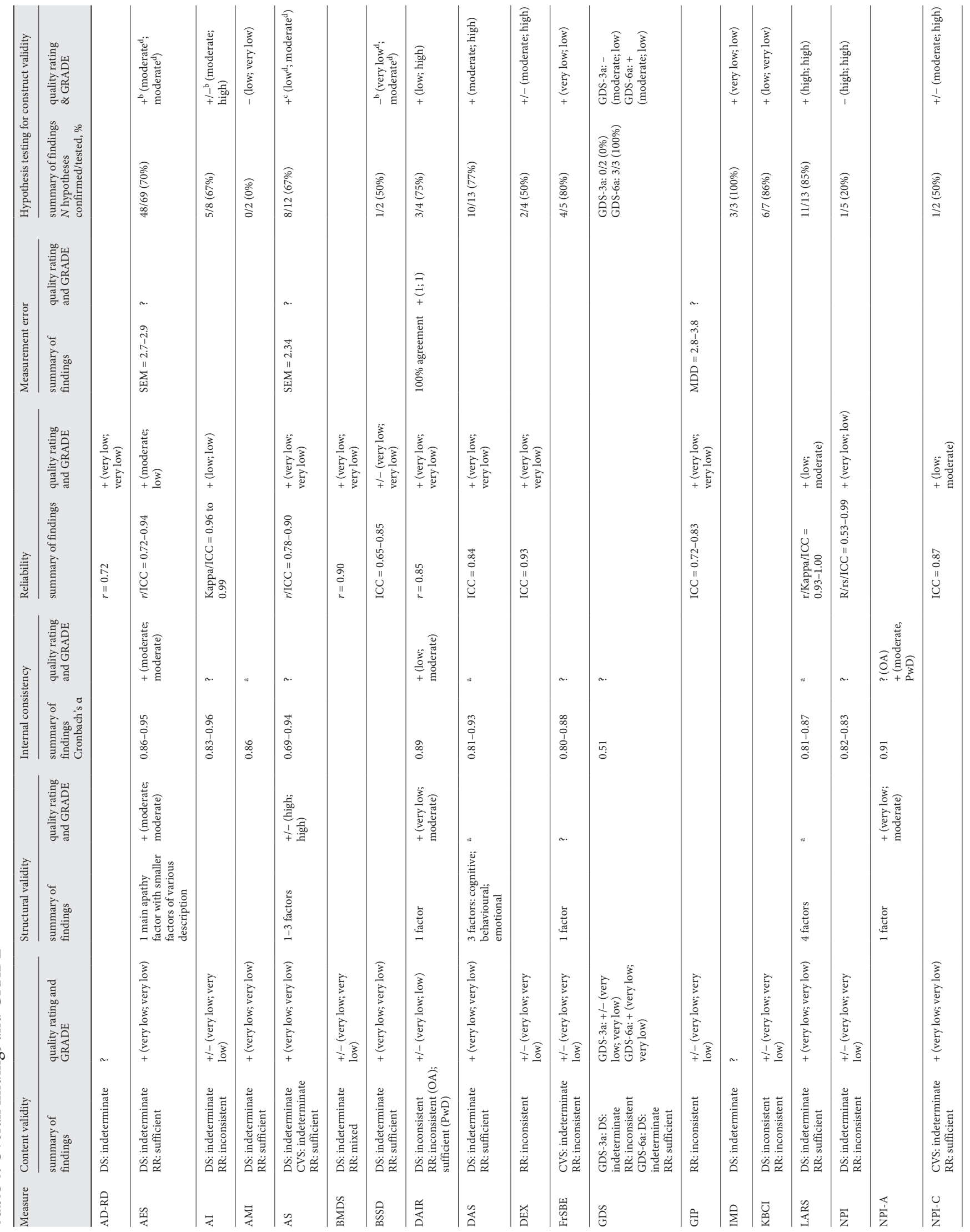




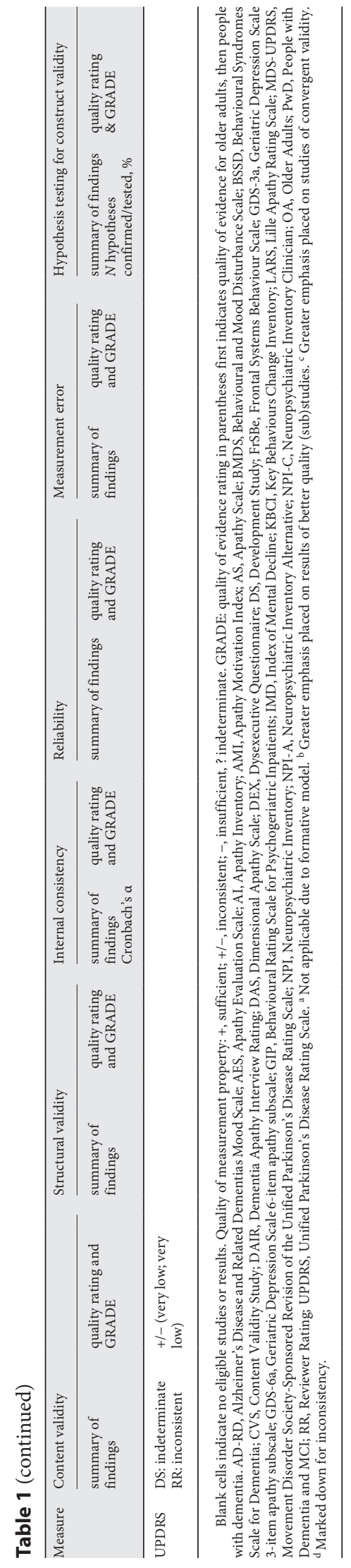

Systematic Review of Apathy Measures in Older Adults and Dementia fied as meeting eligibility criteria (online suppl. Table 1 in Additional File 4). Many publications reported multiple studies, even for the same measurement property, for example, where the measurement property was assessed and reported for different populations or different versions of the same scale or where different methods were used to assess the same measurement property.

The measurement properties and study characteristics are reported in online suppl. Table 2 in Additional File 4. Seven apathy-specific scales were identified: the Apathy Evaluation Scale (AES) [46], Apathy Inventory (AI) [47], Apathy Motivation Index (AMI) [48], Starkstein Apathy Scale (AS) [49], Dementia Apathy Interview and Rating (DAIR) [50], Dimensional Apathy Scale (DAS) [51], and the Lille Apathy Rating Scale (LARS) [52]. Apathy subscales were present in eleven global scales designed to assess a variety of constructs (such as dementia severity and neuropsychiatric symptoms): Alzheimer's Disease and Related Dementias mood scale (AD-RD) [33, 34], Behavioural and Mood Disturbance Scale (BMDS) [35], Behavioural Syndromes Scale for Dementia (BSSD) [36], Dysexecutive Questionnaire (DEX) [37]; Frontal Systems Behaviour Scale (FrSBe) [38], Geriatric Depression Scale (GDS) $[39,40]$, and Behavioural Rating Scale for Geriatric Inpatients (GIP) [41], Index of Mental Decline (IMD) [42], Key Behaviours Change Inventory (KBCI) [43], Neuropsychiatric Inventory (NPI) [44], and Unified Parkinson's Disease Rating Scale (UPDRS) [45]. Only the measurement properties of apathy subscales were assessed, not the overall global scale. Of the publications that met the inclusion criteria, there was one each that pertained to the AD-RD, BMDS, BSSD, DEX, GIP, IMD, KBCI, AMI, and DAIR, two regarding the FrSBe and GDS, 3 regarding the AI and LARS, 4 regarding the UPDRS, 5 regarding the DAS, 8 regarding the AS, 9 regarding the AES, and twelve regarding the NPI. The majority of scales required respondents to select responses from a Likert scale, in relation to various questions or items. Number of scored items in the scales ranged from 1 to 33 . Recall periods ranged from 1 week to 1 month, with some scales not specifying a recall period or specifying since the onset of a disease.

\section{Risk of Bias}

Results of the individual studies and their risk of bias ratings are reported in the online suppl. material (online suppl. Tables 3-5 in Additional File 4). No studies assessed cross-cultural validity, so this is not discussed nor included in the tables. Literature pertaining to development was obtained for all scales except the DEX, GIP, and 
FrSBe. Few additional content validity studies were available that met the eligibility criteria. Most content validity and development studies had indeterminate results, due to a poorly reported or inadequate method. Twenty-seven studies of structural validity across 16 publications met the inclusion criteria $[46,50,53-66]$. Three studies had very good methodological quality, as most studies used exploratory factor analysis or principle component analysis to assess structural validity, instead of the preferred confirmatory factory analysis or item response theory methods. Internal consistency was assessed by 31 publications [36, 46, 47, 49, 50, 53, 55-79] and was considered a valid assessment (i.e. the scale was based on a reflective model) in 38 studies. Some results were indeterminate due to lack of evidence that the scale was unidimensional, and therefore, uncertainty regarding whether internal consistency should apply. There were 38 inter-rater or test-retest reliability studies $[33,35,36,46,47,49,50,53,65,70,73$, $76,77,79-88$ ] from 23 publications. None were of very good methodological quality, and just one was of adequate quality. Methodological quality of reliability studies was mostly limited as a result of not using the optimal statistical method, such as the use of Kappa rather than weighted Kappa, or Pearson or Spearman correlation instead of intraclass correlation coefficient. Where the most appropriate method was used, the model or formula of intraclass correlation coefficient or weighted Kappa was often not reported. Six studies of measurement error were conducted across 4 publications [50, 56, 77, 82]. For all but one study, it was not possible to draw conclusions, due to lack of appropriate anchor-based estimates of minimal important change for any of the scales. One hundred and eighty studies of hypothesis testing for construct (including convergent, divergent, and known group) validity that met the eligibility criteria were found from 45 publications [36, $42,44,46,47,49,50,53-58,60,62,65,67-71,73-75,77-$ $81,83,85,87-100]$. Most reported $p$ values, but not effect sizes, and 21 studies had indeterminate results due to not reporting sufficient information.

\section{Synthesis of Results}

A synthesis of the results of measurement properties per scale, including quality rating and GRADE ratings for older adults and people with dementia, is provided in $\mathrm{Ta}-$ ble 1 .

\section{Apathy-Specific Scales}

Apathy Evaluation Scale

The AES is an 18-item apathy scale based on informant report, self-report (AES-S), or clinician assessment. Nine publications regarding the validity or reliability of the AES met the inclusion criteria. The AES had sufficient content validity, though like other studies, the evidence for this was very low. There was moderate evidence for sufficient hypothesis testing for construct validity and structural validity. The latter result limited the quality of evidence for sufficient internal consistency (Cronbach's $a=0.86-0.95$ ) to moderate also. There was low to moderate evidence for sufficient reliability, except of the AES-S in people with dementia, where test-retest reliability was insufficient. The only measurement property that the AES lacked evidence for was measurement error.

\section{Dimensional Apathy Scale}

The DAS is a 24-item scale, made up of 3 subscales: executive, emotional, and initiation. There is a self-rated and proxy version, and a shorter proxy version (b-DAS), which retains the 3 subscales across just 9 items. Five articles investigating the DAS (including b-DAS) met the inclusion criteria $[62,63,74-76]$. There was very low evidence of sufficient content validity of the DAS, including b-DAS, and sufficient test-retest reliability; however, this evidence came from a single study of the b-DAS, so conclusions may not be generalizable to the full version. There was moderate-to-high-quality evidence of sufficient hypothesis testing for construct validity. Structural validity and internal consistency were not relevant due to this scale's formative nature, and there was no evidence for measurement error.

\section{Lille Apathy Rating Scale}

The LARS was developed to screen for and assess changes in apathy in people with Parkinson's disease and was originally designed as a clinician-rated scale based on observations and answers provided in an interview with the participant. Three articles on the LARS met the inclusion criteria $[65,70,83]$. There was very low evidence of sufficient content validity, low to moderate evidence of sufficient reliability, and high-quality evidence for sufficient hypothesis testing for construct validity. As with the DAS, structural validity and internal consistency were not relevant due to this scale's formative nature, and there was no evidence for measurement error.

\section{Dementia Apathy Interview and Rating}

The DAIR was developed to assess apathy in people with dementia. One article met the inclusion criteria [50]. There was very low evidence for inconsistent content validity of the DAIR in older adults and low evidence for inconsistent content validity in people with dementia. 
There was very low to moderate evidence for sufficient structural validity and low to moderate evidence of internal consistency. There was very low evidence for sufficient test-retest reliability and measurement error and low to high-quality evidence of sufficient hypothesis testing for construct validity.

\section{Apathy Inventory}

The AI is a 3-domain apathy scale, initially created as a self or informant report via face-to-face interview and developed for older adults and people with neurological disorders. Three articles on the AI met the inclusion criteria $[47,72,80]$. Evidence for content validity and hypothesis testing for construct validity was inconsistent. There was low evidence for sufficient reliability and no conclusive evidence for structural validity or internal consistency.

\section{Apathy Scale}

The Apathy Scale (AS) is a 14-item apathy scale, administered through self-report or informant report, via interview. An 11-item paper and pencil version (AS-HC) without subquestions has also been produced [58]. Eight articles on the AS met the inclusion criteria $[49,58-61$, 77, 90, 91]. Despite the high-quality studies, the results regarding structural validity were inconsistent. The ASHC however had moderate to low evidence for sufficient structural validity and internal consistency. There was very low evidence of sufficient content validity and reliability and low to moderate quality evidence for sufficient hypothesis testing for construct validity of the AS. There was no conclusive evidence for internal consistency or measurement error.

\section{Global Scales with an Apathy Subscale}

Neuropsychiatric Inventory

The NPI is a well-known scale for assessing neuropsychiatric symptoms in people with dementia. Each subscale of the NPI represents a different symptom, of which apathy is one. Originally designed as a proxy assessment administered via interview, the NPI now has many variations, including those which score the screening or subquestions, as in the NPI-Alternate (NPI-A) and NPI-Clinician (NPIC). The NPI was the most studied scale, with 12 articles meeting the inclusion criteria $[44,66,73,79,84-88,94-$ 96]. Content validity of the original NPI apathy subscale was inconsistent, as the emotional domain was missing from the screening questions. In contrast, the NPI-C had sufficient content validity. The NPI-A had sufficient structural validity with moderate to very low evidence, and there was moderate evidence for sufficient internal consistency in people with dementia. There was very low to low evidence of reliability for the original NPI. The NPI-C had better evidence of reliability, with low and moderate evidence for sufficient interrater reliability. Hypothesis testing for construct validity was found to be insufficient for the original NPI, supported by high-quality evidence, and for the NPI-C, evidence was inconsistent. The NPI-A lacked conclusive evidence for hypothesis testing for construct validity, and reliability, whilst the NPI-C and NPI had no conclusive evidence for structural validity, internal consistency, or measurement error of the apathy subscales.

\section{Behavioural Syndromes Scale for Dementia}

The BSSD is a measure of neuropsychiatric symptoms, which contains an apathy subscale consisting of 7 items, for which one publication met the inclusion criteria [36]. There was very low evidence for sufficient content validity and inconsistent reliability for face-to-face administration, with insufficient reliability when administered by telephone. There was very low to moderate evidence of sufficient hypothesis testing for construct validity; however, results should be interpreted with caution as no studies of convergent validity were included. There was no conclusive evidence for the remaining measurement properties (structural validity, internal consistency, and measurement error).

\section{Dysexecutive Questionnaire}

The DEX was developed as part of the behavioural assessment of the dysexecutive syndrome test battery. One publication on the DEX met the inclusion criteria [81]. There was inconsistent content validity, very low evidence for sufficient test-retest reliability, and moderateto-high-quality evidence of inconsistent hypothesis testing for construct validity. There was no conclusive evidence for the remaining measurement properties (structural validity, internal consistency, and measurement error).

\section{Scales with Limited Evidence}

The AD-RD, IMD, and UPDRS all had evidence regarding just one measurement property. The $\mathrm{AD}-\mathrm{RD}$ had very low evidence for sufficient test-retest reliability, the IMD had very low to low evidence of sufficient hypothesis testing for construct validity, and the UPDRS had moderate evidence for inconsistent hypothesis testing for construct validity.

There was low to very low evidence of insufficient hypothesis testing for construct validity of the AMI, and whilst there was low evidence for sufficient content valid- 
ity, it is worth noting that some items were too conflated with cognition or disinhibition (e.g., "I get things done when they need to be done, without requiring reminders from others").

The BMDS, FrsBe, GIP, KBCI, and three-item subscale of the GDS all had inconsistent content validity and evidence regarding one other measurement property, although for all cases evidence for content validity came from reviewer ratings only due to absent or indeterminate development and content validity studies. Both the BMDS and GIP had very low evidence for sufficient reliability and inconsistent content validity, with only 55 and $44 \%$ of items relevant to apathy, respectively. Items and response options of the BMDS created confusing double negatives, and the emotional domain of apathy was missing from both the BMDS and the GIP. There was very low to low evidence of sufficient hypothesis testing for construct validity, and in all 3 versions of the FrSBe rated by reviewers, none had the required $\geq 85 \%$ of items relevant to apathy, due to items related to personal hygiene that could be conflated with other impairments. There was moderate to low evidence of insufficient hypothesis testing for construct validity for the three-item subscale of the GDS, and its inconsistent content validity was due to inclusion of items too conflated with physical ability and lack of comprehensiveness. Despite similar inclusion of items that could be conflated with physical ability and dysphoria, the 6-item subscale of the GDS (GDS-6a) had sufficient content validity, as comprehensiveness and comprehensibility were sufficient. The GDS-6a also had moderate to low evidence of sufficient hypothesis testing for construct validity. The KBCI had low to very low evidence of sufficient hypothesis testing for construct validity and inconsistent content validity due to some items not being sufficiently relevant to older adults and people with dementia (e.g., "has a lot of get-up-and-go") and others lacking clear comprehensibility (e.g." "is enterprising"). The results regarding hypothesis testing for construct validity for the IMD, KBCI, GDS-6a, and FrSBe should be interpreted with caution, as no convergent validity studies met the criteria, and convergent validity is a superior indicator of construct validity than divergent or known-group validity [27].

\section{Discussion}

According to COSMIN guidelines, scales should be recommended if they have sufficient content validity, at least low-level evidence for sufficient internal consisten- $c y$, and no high-quality evidence for insufficient properties. The AES, AMI, AS, DAS, GDS-6a, LARS, and NPI-C all had sufficient content validity in older adults and people with dementia, but the AS, GDS-6a, and NPI-C did not have evidence for sufficient internal consistency. The AES had sufficient internal consistency, though the AMI, DAS, and LARS were based on a formative model, so internal consistency was not applicable. Therefore, the AES was the only scale that met the COSMIN criteria for a recommended scale. The (original) NPI was the only scale to meet COSMIN criteria for a scale that should not be recommended for use due to high-quality evidence for insufficient hypothesis testing for construct validity. All other scales could potentially be recommended, depending on further research. However, we argue that the BMDS and GIP are also inappropriate for assessing apathy in older adults and people with cognitive impairment due to inclusion of too many items that are not relevant and conflate apathy with cognition.

This review considered both apathy-specific scales and apathy subscales derived from a global assessment, as though the latter may be designed for screening purposes, as in the NPI, they are often used in place of full assessments, from which conclusions are drawn: for example, the NPI apathy subscale has been recommended as a primary outcome measure in clinical trials [101]. Therefore, it was deemed necessary to assess both types of measures to create a sufficiently comprehensive review of the evidence for all apathy measures available that may be used to assess apathy specifically in people with dementia and older adults. It is worth noting that the best-quality apathy measures were all apathy-specific scales, rather than those derived from a global measure. This highlights the importance of apathy-specific measures and may suggest that apathy subscales derived from global instruments (such as the apathy subscales of the BMDS, BSSD, GDS, IMD, KBCI, UPDRS, and NPI) should not be used to assess apathy in research or clinical practice, unless followed by further assessment. However, there is currently no sufficient evidence to make these conclusions, except for the NPI. The finding that the NPI should not be recommended for assessing apathy contrasts with its popularity and previous recommendations [20, 101]. Our study found that the NPI apathy subscale had insufficient construct validity and inconsistent content validity, suggesting it assesses something other than apathy, which expands previous studies which concluded it had uncertain validity [21]. Whilst this could be due to the low quality of convergent validity studies (which were all of inadequate quality), divergent validity studies also supported 
this finding, as they showed a high correlation with depression, suggesting the NPI apathy subscale may conflate apathy with depression. It is important to note that the NPI was designed as a quick assessment tool for numerous neuropsychiatric symptoms [44], and therefore it is perhaps not surprising that it does not offer a comprehensive and targeted assessment of apathy specifically. Therefore, the NPI may be best used as a screening tool but not as an outcome measure or full clinical assessment of apathy in older adults or people with cognitive impairment.

This systematic review applied a wider search strategy and eligibility criteria than previous systematic reviews $[21,22]$, resulting in the inclusion of a larger number of studies, allowing more evidence to contribute to the results. Despite the numerous studies of measurement properties identified by this review, evidence across all measurement properties was often of low or very low quality. In particular, many development and content validity studies failed to report a systematic process of how items were produced or refined; did not involve patients, carers, or members of the public; or did not provide sufficient detail (e.g., even when it was clear that participants were involved in assessing these properties, it was not clear what aspects [such as items, recall period, instructions, and response options] of the scale participants were consulted about). As such, the included publications offered little evidence for content validity, with all but 2 studies' results being indeterminate, and no study exceeding doubtful methodological quality. As a result, content validity was largely determined entirely by reviewer ratings of the scale itself. COSMIN's reviewer rating technique ensured a validity rating was possible, even in the presence of insufficient information from the development and content validity studies. However, this also meant that content validity conclusions were largely based on very low evidence.

Furthermore, COSMIN guidelines do not advise how to recommend studies of scales based on a formative model, which discounted 3 scales (the AMI, DAS, and LARS) from the recommendations. As such, the COSMIN-guided recommendations of measures are to be taken with caution in this study. Regardless of this, no single scale had overwhelmingly superior measurement properties. The AES, DAS, and LARS all had sufficient content validity, reliability, hypothesis testing for construct validity, and structural validity and internal consistency where applicable, in people with dementia and older adults. The LARS was the scale with the best evidence for good measurement properties and was the only scale to have high-

Systematic Review of Apathy Measures in Older Adults and Dementia quality evidence for at least one measurement property in both older adults and people with dementia. However, the LARS may have less desirable measurement characteristics, as both the self and informant versions involve interviewer ratings, as well as respondent reports, and was the largest scale found by the review, with 33 items assessing apathy, so requires more resources and could be burdensome. The AES had the second most consistent quality evidence across measurement properties and may have preferable measurement characteristics, as there are versions that do not require trained raters and have fewer items. This is consistent with the recommendation of the AES made by others $[20,101]$. The DAS is a promising scale, with good evidence for sufficient measurement properties, with the exception of reliability. The DAS also has desirable measurement characteristics, as the pencil and paper-based scale does not require interviews, and a short version is available.

Previous systematic reviews of apathy measures used QUADAS, which was designed only to assess studies of diagnostic accuracy and applied these to studies of a variety of measurement properties. COSMIN on the other hand provides guidance and criteria for assessing the quality of and evidence for a variety of measurement properties. The high standards set by the COSMIN guidelines and criteria were however sometimes unattainable. For example, in a development study, a lack of justification of recall period and response options can prevent the results of a development study being rated as sufficient, yet these aspects represent a small part of the scale and are rarely provided by even the best quality studies. As COSMIN quality criteria are binary, it risks over simplifying the complexities of the true measurement properties and research evidence. Weighted criteria which place greater emphasis on the items may be preferable for content validity assessment. An alternative for assessing quality of the remaining measurement properties is that used by Radakovic and colleagues [21], which rated each result on a scale of 4-6 possible scores depending on the measurement property being assessed. However, this does not appear to have been developed in a systematic way, unlike COSMIN criteria that were created following a Delphi procedure.

Bias in systematic reviews can be minimized by duplicating all rating activities; however, due to the large number of studies found by this review, this was impractical in this study. The duplication of review for a portion of the included studies did however help discussions around what these flaws may be, limiting subjective decisions. Bias was further minimized by following COSMIN guide- 
lines and creating additional criteria where required, informed by PPI when applicable, that could be followed for all scales.

This review did not restrict the eligibility criteria to people with a diagnosis of dementia or restrict the age criteria to all adults (instead choosing that at least 50\% should meet the criteria). This meant that some studies included participants with various diagnoses, such as Parkinson's disease and depression, and included some participants that were younger than 65 . Therefore, the results may be less applicable to the population we set out to study. However, populations do not neatly segment, and by opting for more liberal inclusion criteria, we were able to include a wide variety of studies that may not otherwise have been included. Furthermore, the GRADE approach to determining the quality of evidence for each measure takes into account the directness of the results, so evidence that was less direct (i.e., studies in other populations) was marked down accordingly.

Apathy is a multidimensional phenomenon, including behavioural, cognitive, social, or emotional domains [1, 102], and so it is expected that comprehensive apathy scales should assess all these aspects of apathy. For this reason, we did not include studies of scales that only assessed a part of the apathy construct, such as studies that investigated the separate subscales of the LARS and DAS. It is possible that the best assessment of apathy is through a combination of scales that assess different individual apathy subdomains, which could be used alongside direct observational methods, such as accelerometers and other experimental methods, that have recently been used to assess certain aspects of apathy such reduced goal-directed behaviour [103]. Future studies could consider the evidence for assessing each individual domain of apathy separately.

\section{Conclusion}

A number of apathy scales of varying quality are available and have been validated in an older adult and dementia community-dwelling population. The development of scales was generally poor, due to lack of transparency and systematic approach in eliciting and refining items and developing the other measurement aspects such as recall period and response options. Future development of scales should include a clear and systematic approach at all stages and involve patients or members of the public as well as professionals to ensure good content validity. The NPI is not recommended for apathy assessment, ex- cept as a screening tool. The LARS has good measurement properties and so is recommended for use in older adults and people with dementia and MCI studies with sufficient resources. The DAS, in particular the resource efficient b-DAS, is a promising scale that requires more research into its properties, particularly reliability. The AES has good measurement properties and characteristics and is recommended for use in older adults and people with dementia and MCI especially in circumstances of limited resources and to limit responder burden.

\section{Acknowledgements}

The authors would like to thank the PPI members Marianne Dunlop, Maureen Godfrey, and Morag Whitworth, as well as the wider "dementia, frail older people, and end of life care" PPI group at the University of Nottingham, for their important insights. We would also like to thank the translators Ester Bellavia, Marta Castro Rodriguez, and Kenichi Sakuda and the senior research librarian Jane Grogan for their contributions.

\section{Statement of Ethics}

As this was a systematic review of published studies, it was not necessary to gain ethical approval.

\section{Conflict of Interest Statement}

The authors have no conflicts of interest to declare.

\section{Funding Sources}

This work forms part of a $\mathrm{PhD}$ project, partially funded by School of Health Sciences and the Division of Rehabilitation, Ageing, and Wellbeing, University of Nottingham. This article also presents independent research funded by the National Institute for Health Research (NIHR) under its Programme Grants for Applied Research Programme (Reference Number RP PG 0614 20007). The views expressed are those of the author(s) and not necessarily those of the NIHR or the Department of Health and Social Care.

\section{Author Contributions}

C.B. conceived of the study with supervision from R.H., S.G., and V.vd.W. C.B. developed and conducted the search and prescreening. C.B., V.vd.W., and Ca.B. screened the studies for eligibility, and C.B., S.G., and V.vd.W. reviewed the included studies for their risk of bias and quality. C.B. drafted the manuscript and all authors edited the text and approved the final manuscript. 


\section{References}

1 Robert P, Lanctôt KL, Agüera-Ortiz L, Aalten $\mathrm{P}$, Bremond F, Defrancesco M, et al. Is it time to revise the diagnostic criteria for apathy in brain disorders? The 2018 international consensus group. Eur psychiatr. 2018 Oct;54:716.

2 Bergh S, Selbæk G. The prevalence and the course of neuropsychiatric symptoms in patients with dementia. Nor Epidemiol. 2012 Nov;22(2).

3 Cipriani G, Lucetti C, Danti S, Nuti A. Apathy and dementia. Nosology, assessment and management. J Nerv Ment Dis. 2014 Oct; 202(10):718-24.

4 Monastero R, Mangialasche F, Camarda C, Ercolani S, Camarda R. A systematic review of neuropsychiatric symptoms in mild cognitive impairment. J Alzheimers Dis. 2009 Jan; 18(1):11-30.

5 Martin E, Velayudhan L. Neuropsychiatric symptoms in mild cognitive impairment: a literature review. Dement Geriatr Cogn Disord. 2020;49(2):146-55.

6 Lam LC, Tam CW, Chiu HF, Lui VW. Depression and apathy affect functioning in community active subjects with questionable dementia and mild Alzheimer's disease. Int J Geriatr Psychiatry. 2007 May;22(5):431-7.

7 Benoit M, Andrieu S, Lechowski L, GilletteGuyonnet S, Robert PH, Vellas B, et al. Apathy and depression in Alzheimer's disease are associated with functional deficit and psychotropic prescription. Int J Geriatr Psychiatry. 2008 Apr;23(4):409-14.

8 Mukherjee A, Biswas A, Roy A, Biswas S, Gangopadhyay G, Das SK. Behavioural and psychological symptoms of dementia: correlates and impact on caregiver distress. Dement Geriatr Cogn Disord Extra. 2017;7(3): 354-65.

9 Sultzer DL. Why apathy in Alzheimer's matters. Am J Psychiatry. 2018 Feb 1;175(2):99100.

10 Armstrong N, Schupf N, Grafman J, Huey ED. Caregiver burden in frontotemporal degeneration and corticobasal syndrome. Dement Geriatr Cogn Disord. 2013;36(5-6): 310-8.

11 Ready RE, Ott BR, Grace J, Cahn-Weiner DA. Apathy and executive dysfunction in mild cognitive impairment and Alzheimer disease. Am J Geriatr Psychiatry. 2003 Apr;11(2):2228.

12 Stuss DT, Van Reekum R, Murphy KJ. Differentiation of states and causes of apathy. The neuropsychology of emotion. New York, NY, USA: Oxford University Press; 2000. p. 340-63.

13 Yeager CA, Hyer L. Apathy in dementia: relations with depression, functional competence, and quality of life. Psychol Rep. 2008 Jun;102(3):718-22.

14 Pickett J, Bird C, Ballard C, Banerjee S, Brayne $\mathrm{C}$, Cowan $\mathrm{K}$, et al. A roadmap to advance dementia research in prevention, diagnosis, in- tervention, and care by 2025. Int J Geriatr Psychiatry. 2018 Jul;33(7):900-6.

15 Onyike CU, Sheppard JM, Tschanz JT, Norton MC, Green RC, Steinberg M, et al. Epidemiology of apathy in older adults: the Cache County Study. Am J Geriatr Psychiatry. 2007 May;15(5):365-75.

16 Chilovi BV, Conti M, Zanetti M, Mazzù I, Rozzini L, Padovani A. Differential impact of apathy and depression in the development of dementia in mild cognitive impairment patients. DEM. 2009;27(4):390-8.

17 Starkstein SE, Jorge R, Mizrahi R, Robinson RG. A prospective longitudinal study of apathy in Alzheimer's disease. J Neurol Neurosurg Psychiatry. 2006 Jan;77(1):8-11.

18 Wadsworth LP, Lorius N, Donovan NJ, Locascio JJ, Rentz DM, Johnson KA, et al. Neuropsychiatric symptoms and global functional impairment along the Alzheimer's continuum. Dement Geriatr Cogn Disord. 2012; 34(2):96-111.

19 Gilmore-Bykovskyi A. Commentary on apathy as a model for investigating behavioral and psychological symptoms in dementia. J Am Geriatr Soc. 2018;66(Suppl 1):S13-6

20 Clarke DE, Ko JY, Kuhl EA, van Reekum R, Salvador R, Marin RS. Are the available apathy measures reliable and valid? A review of the psychometric evidence. J Psychosom Res. 2011;70(1):73-97.

21 Radakovic R, Harley C, Abrahams S, Starr JM. A systematic review of the validity and reliability of apathy scales in neurodegenerative conditions. Int Psychogeriatr. 2015;27(6): 903-23.

22 Mohammad D, Ellis C, Rau A, Rosenberg PB, Mintzer J, Ruthirakuhan M, et al. Psychometric properties of apathy scales in dementia: a systematic review. J Alzheimers Dis. 2018; 66(3):1065-82.

23 Whiting P, Rutjes AW, Reitsma JB, Bossuyt PM, Kleijnen J. The development of QUADAS: a tool for the quality assessment of studies of diagnostic accuracy included in systematic reviews. BMC Med Res Methodol. 2003 Nov;3(1):25.

24 Prinsen CAC, Mokkink LB, Bouter LM, Alonso J, Patrick DL, de Vet HCW, et al. COSMIN guideline for systematic reviews of patientreported outcome measures. Qual Life Res. 2018 May;27(5):1147-57.

25 Terwee CB, Prinsen CAC, Chiarotto A, Westerman MJ, Patrick DL, Alonso J, et al. COSMIN methodology for evaluating the content validity of patient-reported outcome measures: a Delphi study. Qual Life Res. 2018 May;27(5):1159-70.

26 Burgon C, Goldberg S, van der Wardt V, Harwood RH. A systematic review of measures of apathy for older adults: validity, reliability and conceptualisation of apathy. PrAISED Discussion Paper Series. 2019 Jul(5).

27 Mokkink LB, de Vet HCW, Prinsen CAC, Patrick DL, Alonso J, Bouter LM, et al. COS-
MIN risk of bias checklist for systematic reviews of patient-reported outcome measures. Qual Life Res. 2018 May;27(5):1171-9.

28 de Vet HCW, Terwee CB, Mokkink LB, Knol DL. Measurement in medicine: a practical guide. Cambridge: Cambridge University Press; 2011.

29 Park MS, Kang KJ, Jang SJ, Lee JY, Chang SJ Evaluating test-retest reliability in patient-reported outcome measures for older people: a systematic review. Int J Nurs Stud. 2018 Mar; 79:58-69.

30 Terwee CB, Jansma EP, Riphagen II, de Vet HC. Development of a methodological PubMed search filter for finding studies on measurement properties of measurement instruments. Qual Life Res. 2009 Oct;18(8): 1115-23.

31 Mokkink LB, Terwee CB, Patrick DL, Alonso J, Stratford PW, Knol DL, et al. The COSMIN study reached international consensus on taxonomy, terminology, and definitions of measurement properties for health-related patient-reported outcomes. J Clin Epidemiol. 2010 Jul;63(7):737-45.

32 Prinsen CA, Vohra S, Rose MR, Boers M, Tugwell P, Clarke M, et al. How to select outcome measurement instruments for outcomes included in a "Core Outcome Set": a practical guideline. Trials. 2016 Sep;17(1): 449

33 Tappen RM, Williams CL. Development and testing of the Alzheimer's disease and related dementias mood scale. Nurs Res. 2008;57(6): 426-35.

34 Tappen RM, Williams C. Attribution of emotion in advanced Alzheimer's disease: family and caregiver perspectives. Am J Alzheimers Dis. 1998 Sep;13(5):257-64.

35 Greene JG, Smith R, Gardiner M, Timbury GC. Measuring behavioural disturbance of elderly demented patients in the community and its effects on relatives: a factor analytic study. Age Ageing. 1982 May;11(2):121-6.

36 Devanand DP, Brockington CD, Moody BJ, Brown RP, Mayeux R, Endicott J, et al. Behavioral syndromes in Alzheimer's disease. Int Psychogeriatr. 1992;4(Suppl 2):161-84.

37 Burgess PW, Alderman N, Wilson BA, Evans JJ, Emslie H. The dysexecutive questionnaire. Behavioural assessment of the dysexecutive syndrome. Bury St Edmunds, UK: Thames Valley Test Company; 1996.

38 Grace J, Malloy PF. Frontal Systems Behavior Scale (FrSBe): professional manual. Lutz, FL: Psychological Assessment Resources; 2001.

39 Brink TL, Yesavage JA, Lum O, Heersema $\mathrm{PH}$, Adey M, Rose TL. Screening tests for geriatric depression. Clin Gerontol. 1982;1(1): 37-43.

40 Yesavage JA, Brink TL, Rose TL, Lum O, Huang V, Adey M, et al. Development and validation of a geriatric depression screening scale: a preliminary report. J Psychiatr Res. 1982 Jan;17(1):37-49. 
41 Verstraten P, Van Eekelen C. Handleiding voor de GIP: Gedragsobservatieschaal voor de Intramurale Psychogeriatrie. Deventer: Van Loghum Slaterus; 1987.

42 Cucinotta D, Ambrosoli L, Poli A, Martorelli M, Savorani GC, Anzivino F. Clinical assessment of mental decline in elderly people: a proposal for a new quantitative index. Aging. 1995 Feb;7(1):29-34.

43 Kolitz BP, Vanderploeg RD, Curtiss G. Development of the Key Behaviors Change Inventory: a traumatic brain injury behavioral outcome assessment instrument. Arch Phys Med Rehabil. 2003 Feb;84(2):277-84.

44 Cummings JL, Mega M, Gray K, RosenbergThompson S, Carusi DA, Gornbein J. The neuropsychiatric inventory: comprehensive assessment of psychopathology in dementia. Neurology. 1994 Dec;44(12):2308-14.

45 Fahn S, Elton RL; Members of the UPDRS Development Committee. Unified Parkinson's Disease Rating Scale. recent developments in Parkinson's disease, Volume II. Macmillan Healthcare Information; 1987. p. 153-63.

46 Marin RS, Biedrzycki RC, Firinciogullari S. Reliability and validity of the Apathy Evaluation Scale. Psychiatry Res. 1991 Aug;38(2): $143-62$.

47 Robert PH, Clairet S, Benoit M, Koutaich J, Bertogliati C, Tible O, et al. The apathy inventory: assessment of apathy and awareness in Alzheimer's disease, Parkinson's disease and mild cognitive impairment. Int J Geriatr Psychiatry. 2002 Dec;17(12):1099-105.

48 Ang YS, Lockwood P, Apps MA, Muhammed K, Husain M. Distinct subtypes of apathy revealed by the apathy motivation index. PLoS One. 2017;12(1):e0169938-15.

49 Starkstein SE, Mayberg HS, Preziosi TJ, Andrezejewski P, Leiguarda R, Robinson RG. Reliability, validity, and clinical correlates of apathy in Parkinson's disease. J Neuropsychiatry Clin Neurosci. 1992;4(2):134-9.

50 Strauss ME, Sperry SD. An informant-based assessment of apathy in Alzheimer disease. Neuropsychiatry Neuropsychol Behav Neurol. 2002 Sep;15(3):176-83.

51 Radakovic R, Abrahams S. Developing a new apathy measurement scale: dimensional apathy scale. Psychiatry Res. 2014 Nov;219(3): 658-63.

52 Sockeel P, Dujardin K, Devos D, Denève C, Destée A, Defebvre L. The Lille apathy rating scale (LARS), a new instrument for detecting and quantifying apathy: validation in Parkinson's disease. J Neurol Neurosurg Psychiatry. 2006 May;77(5):579-84.

53 Lee SH, Wen MC, Chao CC, Chen YJ, Yen CF. Apathy in late-life depression among Taiwanese patients. Int Psychogeriatr. 2008 Apr; 20(2):328-37

54 Clarke DE, Reekum R, Simard M, Streiner DL, Freedman M, Conn D. Apathy in dementia: an examination of the psychometric properties of the apathy evaluation scale. J Neuropsychiatry Clin Neurosci. 2007;19(1):57-64.
55 Guercio BJ, Donovan NJ, Munro CE, Aghjayan SL, Wigman SE, Locascio JJ, et al. The apathy evaluation scale: a comparison of subject, informant, and clinician report in cognitively normal elderly and mild cognitive impairment. J Alzheimers Dis. 2015;47(2):421-32.

56 Johansson M, Johansson P, Stomrud E, Hagell P, Hansson O. Psychometric testing of a Swedish version of the Apathy Evaluation Scale. Nord J Psychiatry. 2017 Aug;71(6):477-84.

57 Lueken U, Evens R, Balzer-Geldsetzer M, Baudrexel S, Dodel R, Gräber-Sultan S, et al. Psychometric properties of the apathy evaluation scale in patients with Parkinson's disease. Int J Methods Psychiatr Res. 2017;26(4):e1564.

58 Morita H, Kannari K. Reliability and validity assessment of an apathy scale for home-care patients with Parkinson's disease: a structural equation modeling analysis. J Phys Ther Sci. 2016 Jun;28(6):1724-7.

59 Kay DB, Kirsch-Darrow L, Zahodne LB, Okun MS, Bowers D. Dimensions of apathy in Parkinson's disease: exploratory factor analysis of the apathy scale. J Parkinsons Dis. 2012;2(2):161-6.

60 Pedersen KF, Alves G, Larsen JP, Tysnes OB, Møller SG, Brønnick K. Psychometric properties of the Starkstein Apathy Scale in patients with early untreated Parkinson disease. Am J Geriatr Psychiatry. 2012 Feb;20(2):142-

61 Lopez FV, Eglit GML, Schiehser DM, Pirogovsky-Turk E, Litvan I, Lessig S, et al. Factor analysis of the apathy scale in Parkinson's disease. Mov Disord Clin Pract. 2019;6(5):37986.

62 Santangelo G, D’Iorio A, Piscopo F, Cuoco S, Longo K, Amboni M, et al. Assessment of apathy minimising the effect of motor dysfunctions in Parkinson's disease: a validation study of the dimensional apathy scale. Qual Life Res. 2017;26(9):2533-40.

63 Radakovic R, McGrory S, Chandran S, Swingler R, Pal S, Stephenson L, et al. The brief Dimensional Apathy Scale: a short clinical assessment of apathy. Clin Neuropsychol. 2020 Feb;34(2):423-35.

64 Midden AJ, Mast BT. IRT analysis of the frontal systems behavior scale: identifying the best items for use with geriatric patients in primary care. Clin Gerontol. 2017 Aug;40(4):295306.

65 García-Ramos R, Villanueva Iza C, Catalán MJ, Reig-Ferrer A, Matías-Guíu J. Validation of a Spanish version of the Lille apathy rating scale for Parkinson's disease. ScientificWorldJournal. 2014 Oct;2014:849834.

66 Gallo JL, Schmidt KS, Libon DJ. An itemized approach to assessing behavioral and psychological symptoms in dementia. Am J Alzheimers Dis Other Demen. 2009 May;24(2): 163-8.

67 Ang YS, Lockwood PL, Kienast A, Plant O, Drew D, Slavkova E, et al. Differential impact of behavioral, social, and emotional apathy on Parkinson's disease. Ann Clin Transl Neurol. 2018;5(10):1286-91.
68 Carvalho JO. Revision of the frontal systems behavior scale (FrSBe). Doctoral Dissertations. 2011 Jan. p. 1-107. Available from Proquest.

69 Davis TE. The effectiveness of the Geriatric Depression Scale to distinguish apathy from depression in Alzheimer's disease and related dementias. 2008.

70 Dujardin K, Sockeel P, Delliaux M, Destée A Defebvre L. The Lille Apathy Rating Scale: validation of a caregiver-based version. Mov Disord. 2008 Apr;23(6):845-9.

71 Köller L, Knebel M, Haberstroh J, Krause K, Sahlender S, Jakob M, et al. Apathy in dementia care: an investigation in communitydwelling persons using the German informant version of the Apathy Evaluation Scale. GeroPsych. 2016;29(3):129-39.

72 Leone E, Lafont V, Filleau C, Baudu C, Benoit $\mathrm{M}$, Deudon A, et al. Validation de la version soignant de l'Inventaire Apathie. Revue Geriatr. 2008;33(9):777-85.

73 Malakouti SK, Panaghi L, Foroughan M, Salehi M, Zandi T. Farsi version of the neuropsychiatric inventory: validity and reliability study among Iranian elderly with dementia. Int Psychogeriatr. 2012 Feb;24(2):223-30.

74 Radakovic R, Starr JM, Abrahams S. A novel assessment and profiling of multidimensional apathy in Alzheimer's disease. J Alzheimers Dis. 2017;60(1):57-67.

75 Radakovic R, Davenport R, Starr JM, Abrahams S. Apathy dimensions in Parkinson's disease. Int J Geriatr Psychiatry. 2018 Jan; 33(1):151-8

76 Radakovic R, Gray D, Dudley K, Mioshi E, Dick $\mathrm{D}$, Melchiorre G, et al. Reliability and validity of the brief dimensional apathy scale. Arch Clin Neuropsychol. 2020 Jul;35(5):539-44.

77 Serrano-Dueñas M, Martínez-Martín P, Merchán T, Bravo R, Serrano M. Properties of the Apathy Scale (AS) for use on Parkinson's patients. Adv Parkinsons Dis. 2013 May;2(2): 53-7.

78 Stankevich Y, Lueken U, Balzer-Geldsetzer M, Dodel R, Gräber-Sultan S, Berg D, et al. Psychometric properties of an abbreviated version of the Apathy Evaluation Scale for Parkinson Disease (AES-12PD). Am J Geriatr Psychiatry. 2018 Oct;26(10):1079-90.

79 Vilalta-Franch J, Lozano-Gallego M, Hernández-Ferrándiz M, Llinàs-Reglà J, López-Pousa S, López OL. [The Neuropsychiatric Inventory. Psychometric properties of its adaptation into Spanish]. Rev Neurol. 1999 Jul; 29(1):15-9.

80 Stella F, de Andrade LP, Garuffi M, Vital TM, Hernández SS, Ruocco M, et al. Validation of the Brazilian version of the apathy inventory. Int J Geriatr Psychiatry. 2013;28(9):979-86.

81 Shinagawa Y, Nakaaki S, Hongo J, Murata Y, Sato J, Matsui T, et al. Reliability and validity of the Japanese version of the Dysexecutive Questionnaire (DEX) in Alzheimer's disease: validation of a behavioral rating scale to assess dysexecutive symptoms in Japanese patients with Alzheimer's disease. Int J Geriatr Psychiatry. 2007 Oct;22(10):951-6. 
82 Diesfeldt HFA. [Interpreting change scores of the Behavioural Rating Scale for Geriatric Inpatients (GIP)]. Tijdschr Gerontol Geriatr. 2013 Sep;44(4):166-74.

83 Fernández-Matarrubia M, Matías-Guiu JA, Moreno-Ramos T, Valles-Salgado M, Marcos-Dolado A, García-Ramos R, et al. Validation of the Lille's apathy rating scale in very mild to moderate dementia. Am J Geriatr Psychiatry. 2016 Jul;24(7):517-27.

84 Camozzato AL, Kochhann R, Simeoni C, Konrath CA, Franz AP, Carvalho A, et al. Reliability of the Brazilian Portuguese version of the Neuropsychiatric Inventory (NPI) for patients with Alzheimer's disease and their caregivers. Int Psychogeriatr. 2008 Apr;20(2): 383-93.

85 Davidsdottir SR, Snaedal J, Karlsdottir G, Atladottir I, Hannesdottir K. Validation of the Icelandic version of the Neuropsychiatric Inventory with Caregiver Distress (NPI-D). Nord J Psychiatry. 2012 Feb;66(1):26-32.

86 Leung VP, Lam LC, Chiu HF, Cummings JL, Chen QL. Validation study of the Chinese version of the neuropsychiatric inventory (CNPI). Int J Geriatr Psychiatry. 2001 Aug; 16(8):789-93.

87 de Medeiros K, Robert P, Gauthier S, Stella F, Politis A, Leoutsakos J, et al. The Neuropsychiatric Inventory-Clinician rating scale (NPI-C): reliability and validity of a revised assessment of neuropsychiatric symptoms in dementia. Int Psychogeriatr. 2010 Sep;22(6) 984-94.

88 Stella F, Forlenza OV, Laks J, de Andrade LP, Ljubetic Avendaño MA, Gasparetto Sé EV, et al. The Brazilian version of the Neuropsychiatric Inventory-Clinician rating (NPI-C): reliability and validity in dementia. Int Psychogeriatr. 2013 Sep;25(9):1503-11.

89 Valentino V, Iavarone A, Amboni M, Moschiano F, Picillo M, Petretta V, et al. Apathy in Parkinson's disease: differences between caregiver's report and self-evaluation. Funct Neurol. 2018 Mar;33(1):31-5.

90 Guimarães HC, Fialho PPA, Carvalho VA, Santos ELd., Caramelli P, Guimarães HC, et al. Brazilian caregiver version of the Apathy Scale. Dement Neuropsychol. 2009 Dec;3(4): 321-6.
91 Wetmore JB, Arbelo JM, Catalán MJ, Valldeoriola $\mathrm{F}$, Rodriguez-Blazquez $\mathrm{C}$, MartinezMartin P. Psychometric properties of the Apathy Scale in advanced Parkinson's disease. Parkinsons Dis. 2019 Mar;2019:1.

92 Bertens AS, Moonen JE, de Waal MW, FosterDingley JC, de Ruijter W, Gussekloo J, et al. Validity of the three apathy items of the Geriatric Depression Scale (GDS-3A) in measuring apathy in older persons. Int J Geriatr Psychiatry. 2017;32(4):421-8.

93 Belanger HG, Brown LM, Crowell TA, Vanderploeg RD, Curtiss G. The Key Behaviors Change Inventory and executive functioning in an elderly clinic sample. Clin Neuropsychol. 2002 Aug; 16(3):251-7.

94 Choi SH, Na DL, Kwon HM, Yoon SJ, Jeong $\mathrm{JH}, \mathrm{Ha}$ CK. The Korean version of the neuropsychiatric inventory: a scoring tool for neuropsychiatric disturbance in dementia patients. J Korean Med Sci. 2000 Dec;15(6):60915.

95 Politis AM, Mayer LS, Passa M, Maillis A, Lyketsos CG. Validity and reliability of the newly translated Hellenic Neuropsychiatric Inventory (H-NPI) applied to Greek outpatients with Alzheimer's disease: a study of disturbing behaviors among referrals to a memory clinic. Int J Geriatr Psychiatry. 2004 Mar; 19(3):203-8.

96 Kat MG, de Jonghe JF, Aalten P, Kalisvaart CJ, Dröes RM, Verhey FR. [Neuropsychiatric symptoms of dementia: psychometric aspects of the Dutch Neuropsychiatric Inventory (NPI)]. Tijdschr Gerontol Geriatr. 2002 Sep; 33(4):150-5.

97 Weintraut R, Karádi K, Lucza T, Kovács M, Makkos A, Janszky J, et al. Lille apathy rating scale and MDS-UPDRS for screening apathy in Parkinson's disease. J Parkinsons Dis. 2016; 6(1):257-65.

98 Kirsch-Darrow L, Zahodne LB, Hass C, Mikos A, Okun MS, Fernandez HH, et al. How cautious should we be when assessing apathy with the Unified Parkinson's Disease Rating Scale? Mov Disord. 2009 Apr;24(5):684-8.
99 Pedersen KF, Larsen JP, Aarsland D. Validation of the Unified Parkinson's Disease Rating Scale (UPDRS) section I as a screening and diagnostic instrument for apathy in patients with Parkinson's disease. Parkinsonism Relat Disord. 2008 Apr;14(3):183-6.

100 Starkstein SE, Merello M. The Unified Parkinson's Disease Rating Scale: validation study of the mentation, behavior, and mood section. Mov Disord. 2007;22(15):2156-61.

101 Cummings J, Friedman JH, Garibaldi G Jones M, Macfadden W, Marsh L, et al. Apathy in neurodegenerative diseases: recommendations on the design of clinical trials. J Geriatr Psychiatry Neurol. 2015 Sep;28(3): 159-73.

102 Marin RS. Apathy: a neuropsychiatric syndrome. J Neuropsychiatry Clin Neurosci. 1991 Aug;3(3):243-54.

103 König A, Aalten P, Verhey F, Bensadoun G, Petit PD, Robert P, et al. A review of current information and communication technologies: can they be used to assess apathy? Int Geriatr Psychiatry. 2014 Apr;29(4):345-58.

104 Kolitz BP. Development of the Key Behaviors Change Inventory (KBCI), a TBI behavioral outcome assessment instrument. 2000 May.

105 Goetz CG, Fahn S, Martinez-Martin P, Poewe W, Sampaio C, Stebbins GT, et al. Movement Disorder Society-sponsored revision of the Unified Parkinson's Disease Rating Scale (MDS-UPDRS): process, format, and clinimetric testing plan. Mov Disord. 2007 Jan;22(1):41-7.

106 Goetz CG, Tilley BC, Shaftman SR, Stebbins GT, Fahn S, Martinez-Martin P, et al. Movement Disorder Society-sponsored revision of the Unified Parkinson's Disease Rating Scale (MDS-UPDRS): scale presentation and clinimetric testing results. Mov Disord. 2008 Nov;23(15):2129-70.

107 GIP-28 Instruction Manual. [GIP-28 Hanleiding]. [cited 2018 Oct 19]. Available from: https://www.werkenindeouderengeneeskunde.nl/wp - content/uploads/2011/04/GIP-handleiding.pdf. 